\title{
GLI ELEMENTI POPOLARI NELLA LINGUA DI ORAZIO*
}

Partendo dal lavoro di G. Bonfante, La lingua parlata in Orazio, Venosa, 1994, abbiamo condotto un'analisi del metodo di lavoro dell'Autore, attraverso anche apporti personali volti a migliorare e a precisare meglio questioni sopra tutto lessicografiche, legate agli elementi popolari e fami-liari presenti nella lingua delle Satire e delle Epistole.

"Nel 1935 cadeva l'anniversario della nascita di Orazio, ed io [= G. Bonfante] sfogliavo la sua opera pazientemente per ricavarne gli elementi "popolari" '1. Da questo lavoro preparatorio nacque Los elementos populares en la lengua de $\mathrm{Horacio}^{2}$, di cui, proprio sulla rivista fondata dallo stesso Autore, uscirono due parti nel 1936 e un'altra e ultima nel $1937^{3}$, anno in cui, per l'interessamento di un collega spagnolo ${ }^{4}$, fu pubblicato in volume, con una tiratura di pochissimi esemplari ${ }^{5}$.

Nel 1992 "(guarda caso!) [...] anniversario della morte di Orazio"6, G. Bonfante vi ha rimesso mano per seguirne l'edizione italiana7, che appare nel 1994 con il titolo La lingua parlata in Orazio $^{8}$ voluto da chi il libro ha prefato ${ }^{9}$.

Come si giustifica la traduzione di un libro scritto oltre mezzo secolo fa e per di piú senza sostanziali ritocchi e, sopra tutto, aggiornamenti? N. Horsfall risponde, nella utile e brillante Prefazione, ricordando ciò che gli disse, or non è molto, "un illustre ordinario di latino in Inghilterra"10: "contano soltanto i libri; gli articoli non si leggono" 11. Se cosí è, questa edizione rappresenta il primo tentativo di portare all'attenzione degli studiosi ${ }^{12}$ un'opera che è sí stata scritta quando c'era "mancanza non solo di lessici, ma anche di indices verborum per alcuni degli autori piú comuni e conosciuti"13; quando non si avevano a disposizione gli "indici di parole completi, né per Cicerone, né per Livio, né per Ovidio" 14 e non erano piú fortunati "né Pomponio Mela, né Columella, né Seneca, né Quintiliano" ${ }^{15}$; ma che nondimeno ha conservato, al di là di qualche perplessità non soltanto nostra ${ }^{16}$ nel vedere riproposto senza aggiornamenti e aggiustamenti un lavoro di oltre mezzo secolo fa, molto del suo valore ${ }^{17}$. Sia nello specifico, per la messe di dati che raccoglie, analizza, confronta e definisce non soltanto dal punto di vista del latinista, ma anche del romanista e dell'indeuropeista. Sia nel metodo, perché, raccogliendo una sollecitazione di E. Wölfflin ${ }^{18}$, affronta la materia con un approccio diverso da quello seguito da altri studiosi ${ }^{19}$ che hanno lavorato nello stesso campo ${ }^{20}$. "Bisogna prendere una parola, una frase, un'espressione, un fenomeno di Virgilio, di Tibullo o dell' autore che si sta studiando, e seguire la storia di questa parola o di questa espressione o fenomeno in tutti gli altri autori anteriori e posteriori, nelle iscrizioni e persino nelle lingue romanze; potremmo quindi stabilire - ma solo a quel punto - se la parola o l'espressione presa in esame è popolare o volgare o poetica o 
arcaizzante o letteraria o magari semplicemente propria dell'autore, sua peculiare"21. Non ci pare, quindi, di potere perentoriamente affermare che G. Bonfante non abbia distinto "nettamente a livello teorico quale sia di volta in volta il piano sul quale le varie forme in questione sarebbero popolari o volgari senza tenere conto delle variazioni dovute a mutamenti di registro linguistico"22.

E questo modo di procedere ed i risultati che con esso si possono raggiungere cercheremo di mettere in luce attraverso esempi e considerazioni che il libro propone, ma anche attraverso osservazioni e puntualizzazioni scaturite dalla sua lettura.

Gli elementi popolari che si trovano specialmente nelle Satire e nelle Epistole di Orazio $^{23}$ avevano già attirato l'attenzione di diversi studiosi ${ }^{24}$, tra cui, sia pure con risultati molto diversi, E. Wölfflin ${ }^{25}$, F. Ruckdeschel ${ }^{26}$ e J. Bourciez ${ }^{27}$. Tuttavia G. Bonfante ${ }^{28}$, pur senza trascurare i predecessori, anzi rendendo loro merito quand'era il caso $^{29}$, ha individuato ed esplorato un aspetto estremamente ricco, ma che stranamente era stato trascurato.

La lingua delle Satire e delle Epistole, ci rivela, se indagata a fondo, tutta un'altra lingua latina, una lingua latina che viveva nel popolo a fianco della lingua letteraria e raffinata dei Cesari e dei Taciti. Una lingua in cui si diceva caballus per equus, comedō per edō, cantō per canō, bellus per pulcher, auricula per auris, bucca per gĕna, auscultō per audiō, uâdō per eō, curtus per mutilus, fricō per terō, siccus per aridus, lassus per fessus, spissus per densus, portō per ferō, serpēns per anguis, prömittō per polliceor, gemellus per geminus, dorsum per tergum, capsa per scrinium, ecc. Una lingua in cui esistevano parole come charta, crepō, ambulō, captō, scabō, conpîlo, crassus, trascurate tutte dai buoni autori.

Si può vedere, dunque, anche attraverso i pochi esempii ${ }^{30}$ scelti, che questa lingua, parlata a Roma ai tempi di Orazio, si avvicina moltissimo alle lingue romanze e cosí, il grande poeta latino diventa una fonte preziosa per lo studio delle lingue vive di Spagna, di Francia e d'Italia. Infatti, molte espressioni da lui usate vivono ancora oggi sulla bocca della gente di Madrid, di Parigi e di Roma e tante di quelle parole, che erano usate dal popolo nella conversazione quotidiana o avevano una connotazione volgare al tempo di Orazio, si sono conservate, vuoi nella stessa funzione, vuoi nello stesso valore.

Non si possono studiare gli elementi popolari in Orazio, senza farsi prima un'idea di quelli presenti negli altri autori. E, pertanto, senza ripetere le idee fondamentali già espresse da altri studiosi, a cominciare da E. Wölfflin ${ }^{31}$, riassumeremo soltanto i risultati piú importanti raggiunti dalla ricerca sul lessico degli autori latini, sopra tutto dal punto di vista statistico.

Gli elementi popolari sono molto frequenti nella lingua della commedia, sopra tutto in Plauto; nei quattro poeti satirici, cioè, in Orazio, Lucilio, Persio e Giovenale; in Marziale e Catullo; in Cicerone, principalmente quello delle Lettere ${ }^{32}$; in Petronio. Seguono gli scrittori tecnici che, poco preoccupati dell'aspetto stilistico, hanno tramandato una lingua semplice e assai vicina al linguaggio quotidiano: Varrone, Catone, 
Columella (agronomi); Celso (medico); Vitruvio (architetto); Plinio il Vecchio e Seneca (naturalisti). Anche la lingua dei poeti elegiaci (Tibullo, Properzio, Ovidio), piuttosto familiare e poco solenne, pur contenendo un numero discreto di elementi popolari, ne ha, naturalmente, molto meno di quella dei satirici, per esempio, o dei comici. Invece, tra gli autori più 'aristocratici' e dunque meno 'popolari', per usare, qui come altrove, la terminologia di G. Bonfante, bisogna citare almeno i cinque epici (Lucano, Valerio Flacco, Silio Italico, Stazio e, prima di tutti, Virgilio) ${ }^{33}$. I prosatori piú aristocratici, piú artificiali, piú lontani dalla lingua parlata sono, ovviamente, gli storici. È fuori di dubbio, infatti, che Sallustio, Cesare, Tito Livio, Tacito siano i quattro grandi puristi romani. Tacito, specialmente, è quasi impermeabile ai volgarismi, confermando cosí sia il giudizio di Plinio il Giovane, che definiva il suo stile $\sigma \varepsilon \mu v \omega^{34}$ e sia quello ch'egli stesso si rivolgeva, quando attribuiva alle sue opere il carattere della

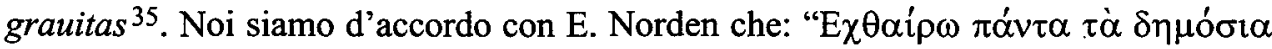
risuona da ogni suo periodo"36.

Questa diversità di registri si coglie anche all'interno dell'opera di Orazio. Dopo le Odi, infatti, che sono di stile molto elevato, vengono, in ordine discendente, gli Epodi, l'Arte Poetica - ancora molto letteraria -, le Epistole e infine le Satire, che si sostanziano di elementi popolari.

A questo punto, però, s'impone una riflessione.

Non c'è dubbio che sia esistita "una lingua volgare latina, profondamente diversa dalla lingua dell'aristocrazia romana" 37 , che è quella dei prosatori piú importanti. Tuttavia non bisogna credere - come anche questo libro di G. Bonfante ci ammonisce che il vocabolario latino si divida tout court in 'aristocratico' e in 'popolare'. Perché se è vero che esiste la lingua oscena, la lingua volgare, la lingua familiare, la lingua della conversazione; la lingua del forum, dei tribunali, delle balineae; la lingua della prosa storica e filosofica, la lingua dell'epos e dell'elegia, ecc. È altrettanto vero, però, che tutte queste varietà di linguaggio s'incrociano e si mescolano, si sovrappongono, si oppongono e si fondono. Pertanto, questo bisogno di classificazione forse indispensabile per il nostro spirito, taglia invece quel fenomeno vivo e variegato che è la lingua e ci dà di essa, necessariamente, un'idea imperfetta $\mathrm{e}$ incompleta. Tuttavia siamo anche noi convinti che "sotto tutte queste differenze secondarie, fluttuanti ed a volte accidentali, resta la grande, profonda differenza di classe: quella che separava il popolo di Roma, la plebe, gli schiavi, dall'aristocrazia romana, dai senatori, dai cavalieri, dai funzionarii" 38 .

Ma passiamo ad interrogare il testo di Orazio $^{39} \mathrm{e}$ a vedere ciò che si può dire sugli aspetti popolari, familiari o volgari della lingua latina. E lo faremo scegliendo qualche esempio, dei molti presenti nel libro, per mostrare come ha lavorato G. Bonfante, senza precluderci, nel contempo, qualche intervento per migliorare o precisare qualche particolare.

Ambŭläre ${ }^{40}$ è un verbo che compare tre volte nelle Satire ${ }^{41}$ e negli Epodi ${ }^{42}$, mentre non viene utilizzato nelle $O d i$. 
Il carattere popolare - non volgare - di questo verbo appare con chiarezza dal fatto che è "una parola attentamente evitata dagli storici e dagli epici" 43 . È completamente assente, infatti, in Lucilio, Virgilio, Lucano, Valerio Flacco, Silio Italico e Stazio ${ }^{44}$. E, tra $\mathrm{i}$ primi, manca del tutto in Cesare e Sallustio ${ }^{45}$, mentre in Tacito ${ }^{46}$ e Tito Livio ${ }^{47}$ non è attestato che una volta soltanto e, nel secondo, per di piú, in un discorso. Anche Ovidio $^{48}$, Columella ${ }^{49}$ e Properzio ${ }^{50}$ non ne hanno che due esempii, ma "non sono da trascurare ${ }^{51]}$ "52, specialmente quelli di Properzio ${ }^{53}$.

Il verbo è, al contrario, molto frequente in Plauto (43 esempii), Cicerone (38 esempii), Celso (26 esempii), Seneca (27 esempii) e Plinio il Vecchio (18 esempii) ${ }^{54}$. Mentre la sua assenza "in autori i cui testi sono brevi come Lucilio, Catullo e Persio, non ha una grande importanza e può essere casuale" 55 .

Se il carattere popolare della parola è sufficiente per spiegare la sua frequenza in Plauto e quello tecnico - clinico in questo caso - la sua frequenza in Celso, tali motivazioni non giustificano la sua presenza in scrittori come Seneca, Plinio il Vecchio e, sopra tutto, Cicerone. In quest'ultimo, infatti, il verbo non si trova soltanto 4 volte nelle Lettere ${ }^{56}$ - il che non ci stupirebbe piú di tanto ${ }^{57}$ - ma appare altresí 19 volte nelle Opere filosofiche ${ }^{58}$ e 4 volte nelle Orazioni ${ }^{59}$. Ci sembra chiaro che l'influenza del gr. $\pi \varepsilon \rho \imath \pi \alpha \tau \omega$ dev'essere tenuta presente (basta pensare alla scuola peripatetica), la quale, per cosi dire, ha nobilitato questo verbo e l'ha fatto entrare nel vocabolario filosofico. La parola ambülāre ci mostra bene la complessità di questi studii di lessicografia: ogni parola ha la sua storia ed è impossibile fissare delle leggi, del tipo delle leggi fonetiche. La parola segue sempre la cosa, l'idea, l'azione che rappresenta; per questo ci sono idee, cose, azioni che sono volgari o popolari per la loro stessa natura e ce ne sono che, proprio per il loro carattere tecnico o altro, sono limitate ad una certa categoria di autori ed evitate da altri. Se non si tenesse conto di ciò, non si riuscirebbe a capire perché un poeta epico avrebbe dovuto usare con una certa frequenza un verbo indicante un'azione familiare, come ambŭlāre, quando aveva a disposizione il classico ire. Di conseguenza, perché una classificazione degli autori in 'popolari' e 'aristocratici' non può essere fatta soltanto sulla base di parole che hanno il valore di ambulare, senza negare, per altro, che il verbo fornisce, anche da questo punto di vista, elementi interessanti.

En passant, si potrebbe chiedere ai romanisti se il carattere sicuramente popolare di questo verbo non debba forse invitarci a porre, ancora una volta, il problema dei rapporti tra il lat. ambülāre, l'it. andare e il fr. aller ${ }^{60}$, che ieri come oggi si configura come "uno dei problemi più oscuri della linguistica romanza"61.

L'inconveniente cui abbiamo accennato, cioè l'assenza della parola per ragioni tecniche e non stilistiche, si può in parte evitare se si prendono in considerazione i sinonimi. Ci sono, infatti, coppie di parole di cui una è popolare, persino volgare, l'altra è piú raffinata, letteraria; talvolta l'opposizione è tra la prosa e la poesia e cosí di seguito. È ben vero che non è sempre facile dimostrare che due parole sono equivalenti dal punto di vista del significato, ciò nonostante ci sono indici che possono essere considerati sufficienti. Per esempio, quando un glossatore glossa con la parola A la paro- 
la B; quando la parola A si usa nelle stesse frasi, negli stessi passi, negli stessi proverbi della parola $B$; quando si vede chiaramente che la parola $A$ può sostituire quella $B$. Un esame della frequenza di due parole ci può dare talvolta risultati interessanti, anche se le due parole non sono del tutto sinonimi. Non è necessario che la parola $\mathrm{A}$ sostituisca la parola $\mathrm{B}$ in tutti i suoi usi: si terrà conto soltanto di quegli usi in cui $\mathrm{B}$ può essere sostituito da A.

Ecco alcuni esempii. ambülāre

Anche se una volta sola e per di piú in un discorso, Orazio usa defricāre ${ }^{62}$, il verbo frícāre e i suoi composti sono popolari; anzi, il semplice ha anche un significato osceno, che è rimasto nell'italiano, dove fregare è in generale poco elegante e si preferisce strofinare. Frĭcäre manca completamente negli storici, nei poeti epici e anche in Cicerone e negli elegiaci. Lo si trova invece, sia pure con una frequenza bassissima, in Plauto ( 5 volte) ${ }^{63}$, in Catone, in Catullo, nei poeti priapei, in Petronio, in Columella, in Giovenale (1 volta) ${ }^{64}$. La sua presenza - rispettivamente una e due volte ${ }^{65}$ - nelle opere di scienze naturali di Seneca ${ }^{66}$ e di Plinio il Vecchio ${ }^{67}$ si spiega, evidentemente, come un tecnicismo. I composti sono meno proscritti invece, forse perché in essi si stempera quel carattere marcatamente osceno che aveva la forma semplice ${ }^{68}$, tanto che arrivano a penetrare in Cicerone ${ }^{69}$, in Svetonio ${ }^{70}$, in Quintiliano ${ }^{71}$ e, sia pure piú tardi, negli elegiaci ${ }^{72}$. Ora, nel caso di fričāre, abbiamo un sinonimo 'aristocratico' perfetto $^{73}$ in terěre ${ }^{74}$, che si trova nei migliori autori. E, come di norma avviene, terěre scompare nelle lingue romanze ${ }^{75}$, mentre fricāre è quanto mai vivo ${ }^{76}$, perché queste tendono a continuare la parola in uso presso il popolo e non viceversa.

Un'altra coppia di sinonimi molto interessante è rappresentato da dēsipěre e furěre su cui $\mathrm{G}$. Bonfante invece si sofferma poco ${ }^{77}$. L'identità delle due parole è provata, per esempio, dal confronto tra questi due passi delle Odi: "recepto // dulce mihi furere est amico"78 $\mathrm{e}$ "dulce est desipere in loco"79. Il loro utilizzo, però, è tutt'altro che omogeneo negli autori latini. Infatti, mentre furěre compare in tutti, désipěre è sí evitato da Sallustio, da Cesare, da Tito Livio e da Tacito ${ }^{80}$, ma lo si trova in Plauto ${ }^{81}$, in Cicerone $^{82}$, in Giovenale 83 , in Celso ${ }^{84}$, in Seneca ${ }^{85}$, in Aulo Gellio ${ }^{86}$, in Apuleio ${ }^{87}$, in Lucrezio $^{88} \mathrm{e}$ in autori cristiani ${ }^{89}$.

Non è facile, comunque, trarre qualche indicazione sicura su desipere che Orazio usa quattro volte, di cui una in quell'opera di alto rigore stilistico che sono le Odi ${ }^{90}$. Tuttavia, un esame della sua diffusione sembra portare alla conclusione che la lingua letteraria cerchi di evitarla, per cui crediamo che si possa accettare il giudizio formulato da G. Bonfante: "sembra un verbo popolare" 91 .

Per l'altro equivalente, dēlīrāre ${ }^{92}$, che compare due volte nelle Epistole ${ }^{93}$, ma manca nei puristi, fatta eccezione per Cicerone ${ }^{94}$ e negli epici, con una sola presenza in Lucrezio ${ }^{95}$, può forse valere lo stesso giudizio appena espresso.

La differenza tra edĕre e comĕdĕre ${ }^{96}$ è piú netta: comĕdĕre che s'incontra due volte nelle Epistole ${ }^{97}$, è la forma popolare di eděre, per questo non è sicuramente un caso che "i due brani in cui comedō appare in Orazio siano in discorso diretto" 98. 
Coměderre, che è già frequente in Plauto (26 occorrenze) $)^{99}$, in seguito si trova in Terenzio (2 volte contro $\left.1^{[100]}\right)^{101}$, in Varrone $(5 \text { contro } 1)^{102}$, in Cicerone (13 contro $10)^{103}$, in Seneca (3 contro 30[104] $)^{105}$, in Columella ( 1 contro 0$)^{106}$, in Petronio (10 contro 3$)^{107}$. Ancora in Tertulliano comĕdĕre è raro (16 volte, ma 7 in loci biblici $)^{108}$, mentre il verbo usuale per 'mangiare' è eděre ( 37 volte, seppure 13 in loci biblici $)^{109}$. Nella Vulgata, però, il trionfo di comědĕre è assicurato: 535 esempii nell'Antico Testamento (e 15 nel Nuovo), contro i 27 di edĕre (18 nel Nuovo) ${ }^{110 !}$

In generale, tutto il problema dei verbi che significano 'mangiare' in latino è assai complesso ${ }^{111}$ e non è questo il luogo per affrontarlo. Non possiamo, però, non notare che, quando Svetonio riporta frasi di Augusto, gli fa dire una volta "gustauimus"112, una volta "comedi"113, una volta "manducaui"114. Ma lo stesso Svetonio, quando in quei medesimi passi parla dell'imperatore, usa il verbo piú letterario "uescebatur"115. $\mathrm{Se}$, dunque, "all' epoca di Augusto edo era [già] scomparso dalla lingua volgare e forse anche da quella parlata in generale"116, sostituito da coměděre, Orazio ci offre qui un aperçu interessante della lingua della conversazione del suo tempo.

Al posto di eděre, nell'area romanza sopravvive coměděre nella Penisola Iberi$\mathrm{ca}^{117}$, ad esclusione della Catalogna, che ha manducare ${ }^{118}$, come la Gallia ${ }^{119}$, la Sardegna $^{120}$, l'Italia ${ }^{121}$, la Dalmazia e la Romania ${ }^{122}$.

Passiamo ora ad esaminare altre coppie di sinonimi, ma di tipologia un po' diversa, perché in esse manca l' opposizione tra un elemento del parlare quotidiano ed uno letterario. Qui, infatti, una delle due parole è di uso corrente; è, cioè, per cosí dire, indispensabile ad ogni utente della lingua ed è, quindi, frequente in tutte le classi sociali $\mathrm{e}$ in tutti i generi letterari. L'altra, invece, è o una parola popolare, evitata da tutti i buoni autori, oppure una parola letteraria, sconosciuta al popolo.

Per esempio, cŭtis ${ }^{123}$ che si trova 3 volte nelle Epistole ${ }^{124}$ ed una nelle Odi ${ }^{125}$, è una parola quasi sinonima di pěllis, benché non si possa negare che una differenza 'di classe' tra le due ci sia. Infatti, cŭtis manca in Sallustio, in Cesare, in Tacito, in Cicerone, in Tibullo, in Virgilio, in Lucrezio e in Terenzio ${ }^{126}$ e non compare con una certa frequenza che in Giovenale ${ }^{127}$, in Ovidio ${ }^{128}$, in Seneca ${ }^{129}$, in Marziale ${ }^{130}$, in Apuleio ${ }^{131}$ e nella Vulgata ${ }^{132}$, mentre, per contro, si trovano esempii straordinariamente numerosi in Celso ${ }^{133}$ e in Plinio il Vecchio ${ }^{134}$, senza dubbio a causa di un suo uso tecnico, che ha conservato in alcuni dialetti italiani $1^{35}$. Pěllis, invece, è una parola che nessuno scrittore evita ${ }^{136}$ e resta perfettamente vivo nelle lingue romanze ${ }^{137}$. La differenza tra cŭtis e pěllis, dunque, non è della stessa natura di quella riscontrata nelle coppie precedentemente studiate.

Il caso di uŏcāre clämāre ${ }^{138}$ è l'opposto del precedente. Uŏcāre è il verbo corrente. Clāmāre ${ }^{139}$, pur evitato da Orazio, nelle Odi e negli Epodi, da Cesare, da Sallustio e da Tacito ${ }^{140}$ è però frequente in Plauto ${ }^{141}$, in Stazio ${ }^{142}$, in Ovidio ${ }^{143}$, in Commodiano ${ }^{144}$, e s'incontra "saepissime" 145 in Cicerone, nella Vulgata e negli scrittori cristiani. Nell'opera di Orazio, clāmāre è attestato tre volte nelle Satire ${ }^{145}$ ed una nelle Epistole ${ }^{146}$. Non soltanto. Il nostro poeta fa di clāmāre un uso del tutto speciale, 
molto raro nella latinità e sicuramente popolare: lo costruisce nel significato di $u \check{c} c \bar{a}-$ re, preferito dai classici, con il doppio accusativo ${ }^{147}$. Va da sé che clāmāre sopravvive

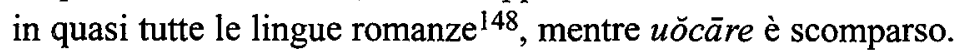

Abbiamo detto che quella delle Odi è una lingua pura, in cui non vi è traccia di parole popolari, ma non bisogna esagerare. Non si deve pensare cioè di trovare nelle Odi la pietra di paragone, il talismano per separare ciò che è proprio della lingua di un Cesare o di un Tacito, da ciò che non vi appartiene. Questa pietra di paragone, questo talismano non esiste da nessuna parte; ogni parola pone un problema diverso, che bisogna risolvere faticosamente, per tentativi, per ipotesi e che, talvolta, purtroppo, può capitare di non risolvere affatto.

Prendiamo, per esempio, crĕpäre ${ }^{149}$.

Nessuno dubita che questo verbo, evitato dai buoni prosatori, tra cui Cesare e Cicerone ${ }^{150}$, ma di una certa frequenza in Plauto ${ }^{151}$ e che s'incontra "saepe apud comicos" e "saepius"152 presso gli scrittori cristiani, sia piuttosto popolare ${ }^{153}$. Ciò nonostante, lo si trova una volta, nelle Satire e nelle Epistole ${ }^{154}$ : il che è normale; ma anche due volte nelle $O d i^{155}$ ed una volta sia negli Epodi sia nell'Arte Poetica ${ }^{156}$ : il che non lo è affatto. La nostra sorpresa però diminuirà, quando constateremo che Virgilio e Persio ne hanno 3 esempii ${ }^{157}$, Stazio $11^{158}$, Lucano e Properzio 2 ciascuno ${ }^{159}$. Forse si tratta di una parola evitata dai prosatori, ma non dai poeti; o almeno i poeti sembrano farne un uso molto meno accurato degli scrittori in prosa 160 .

Un altro caso simile è rappresentato da spissus ${ }^{161}$, il cui equivalente 'aristocratico' è densus.

Spĭssus non compare nei buoni prosatori. Infatti, manca in Cesare, in Sallustio, nelle Opere filosofiche e nei Discorsi di Cicerone e in Tacito ${ }^{162}$. Si trova però 3 volte in Plauto ${ }^{163}, 2$ nelle Lettere di Cicerone ${ }^{164}, 10$ in Seneca ${ }^{165}, 4$ in Petronio ${ }^{166}, 12$ in Columella $^{167}, 2$ in Quinto Rufo ${ }^{168}, 9$ in Ovidio $^{169}$ e poi ancora negli epici ${ }^{170}$ e nella Vulgata ${ }^{171}$. Spissus è presente anche in Cecilio Stazio, Titinio, Turpilio, Varrone ${ }^{172}$. Questo aggettivo appartiene, dunque, come la parola precedente, al registro popolare e poetico nello stesso tempo: come parola popolare entra nelle Epistole ${ }^{173}$ e come parola poetica trova accesso nelle $O d i^{174}$. Una conferma della nostra analisi ci viene dalle lingue romanze, in cui spissus sopravvive quasi dappertutto ${ }^{175}$, mentre densus è pochissimo rappresentato 176 .

Altre volte, invece, soltanto la statistica ci può dare un'idea dello stato reale della questione. $\grave{E}$ il caso ben noto di grandis e magnus ${ }^{177}$. La differenza di significato ${ }^{178}$ - che per altro non è sempre nettamente percepibile - è ben presto scomparsa e, in ogni caso, restano molti gli esempii comuni. Ora, grandis si trova in tutti i migliori autori: Cesare ne ha 3 esempii ${ }^{179}$, Tacito $14^{180}$, Cicerone ne ha molti ${ }^{181}$, di cui 37 nei Discorsi ${ }^{182}$ e 13 nelle Opere filosofiche ${ }^{183}$, Virgilio 10 di cui 3 nell' Eneide ${ }^{184}$. '̇ chiaro che grandis non è un termine volgare e neppure popolare, tant'è che non subisce alcun ostracismo da parte degli scrittori puristi. Tuttavia, se si considera che Plauto ha 21 grandis $^{185}$, Marziale $43^{186}$, Persio - la cui opera è però molto breve $-8^{187}$, Giovenale $22^{188}$, non 
si potrà negare che la lingua popolare manifesti una preferenza assai chiara per grandis che, nelle lingue romanze, rimpiazzerà quasi dappertutto magnus ${ }^{189}$. Saranno sufficienti, crediamo, due esempii tratti dalle stesse Satire, per evidenziare come grandis sia riservato da Orazio ai passi piú familiari, mentre quando lo stile si fa solenne e quasi epico la preferenza del poeta vada a magnus. Basta confrontare quel dimesso: "filius uxorem grandi cum dote recuset"190 con l'aulico distico: "nec quod auus tibi maternus fuit atque paternus // olim qui magnis legionibus imperitarent"191, per rendersene conto.

Da quanto detto, dunque, apparirà del tutto naturale trovare nell'opera di Orazio ${ }^{192}$ 5 grandis nelle Odi e negli Epodi e 8 nelle Satire e nelle Epistole, contro, rispettivamente, 21 e 67 magnus ${ }^{193}$.

Consideriamo ancora caněre e cantāre ${ }^{194}$. Il secondo si trova in tutti gli autori puristi ${ }^{195}$, ma è sicuramente meno raffinato di caněre, tanto che si è conservato nelle lingue romanze ${ }^{196}$. Un'altra conferma ci viene da Plauto, che ha 5 cantäre contro 1 solo canĕre ${ }^{197}$ e da Persio, che non conosce che il primo ( 5 esempii ${ }^{198}$. Non sarà sorprendente, dunque, trovare nelle Odi e negli Epodi 10 canère e 6 cantāre (piú 1 nel Carmen saeculare), mentre le Satire hanno 5 caněre e le Epistole 2 (piú 1 nel Carmen saeculare) contro, rispettivamente, 6 cantāre e 1 (piú 1 nel Carmen saeculare). ${ }^{199}$ Benché la differenza non sia grande, crediamo che l'indicatore di tendenza sia chiaro. D'altra parte, basta leggere, p. es., il breve passo delle Satire, "[...] Pollio regum // facta canit pede ter percusso [...]"200, per rendersi conto quanto sarebbe difficile usare cantare, quando lo stile si fa epico, come in questo passo. Un simile verbo, invece, è pienamente al suo posto, quando dice dei cantores professionisti "ut numquam inducant animum cantare rogati" 201 .

Senza continuare questa esemplificazione, crediamo che studiando tutto ciò che l'arte di Orazio ha di vivo, di popolare, di parlato, si arriva a toccare il punto piú delicato e direi simpatico della sua poesia. Infatti, facendo rivivere in Roma il genere abbandonato della satira, Orazio ha dato alla letteratura latina il gioiello forse piú bello e, senza dubbio, piú originale, tanto da fare dire a Quintiliano 202 che "satura quidem tota nostra est". E noi, con lo studio degli elementi popolari e familiari di queste composizioni, entriamo direttamente nella vita quotidiana degli antichi Romani e passiamo qualche piacevole ora, partecipando alle conversazioni amicali di Orazio e dei suoi contemporanei. Ma con la lingua delle Satire e delle Epistole, Orazio non soltanto ci ha aperto uno scorcio unico e toccante sul mondo romano, ma ha conseguito anche quell'obiettivo, che è forse il piú difficile da raggiungere nella poesia: l'arte del semplice.

\section{APPENDICE}

Abbiamo ritenuto di relegare in un'Appendice i rilievi che si possono muovere alla traduzione, a certe scelte editoriali e alla correttezza formale ${ }^{203}$. A questa decisione siamo stati spinti dal fatto che, come vedremo, sono davvero tanti, specialmente quel- 
li che si riferiscono agli ultimi due punti, benché le recensioni ${ }^{204}$, sotto qualunque taglio si presentino, non ne facciano quasi parola, con l'unica eccezione per quella di A. Traina ${ }^{205}$. Un lavoro, quest'ultimo, che "i modesti contributi di un filologo", come scrive, non senza qualche compiacimento l'Autore ${ }^{206}$, rendono invece importante, al punto che lo si deve considerare un'integrazione indispensabile di BONFANTE 1994. Ma che altresí precisa e corregge riferimenti bibliografici presenti nella Prefazione di N. Horsfall ${ }^{207}$ e non soltanto ${ }^{208}$; che segnala l'aggiunta e la risistemazione di alcuni lemmi rispetto all'edizione spagnola ${ }^{209}$ e qualche refuso ${ }^{210}$.

Passiamo ora al testo di G. Bonfante, dividendo le nostre osservazioni secondo quanto indicato nella $\mathrm{n} .1$.

A) La traduzione è in genere scorrevole, senza errori e fraintendimenti gravi, ma le imprecisioni che s'incontrano non sono poche. Qualche esempio soltanto: non "saggi", ma "lavori" (p. 23, r. 2: sp. trabajos); "trascurare" è forse meglio di "disprezzare" (p. 37, r. 25: sp. despreciar); non "sta cambiando", ma "è alquanto consumato" (p. 37, r. 37: sp. está algo gastado) sotto il profilo della specificità semantica; non "stanza permanente", ma "abitazione permanente" (p. 53, r. 27: sp. habitación estable); non "si veda", ma "si comparino" (p. 71, n. 46: sp. compárense); non "brani", ma "passi" (p. 123, r. 19: sp. pasajes); non "molto ben delimitato", ma "ben delimitato" (p. 123, r. 27: sp. bien delimitado); ecc.

B) Per quanto concerne certi interventi sulla sistemazione della materia, dobbiamo subito dichiarare, che non siamo stati in grado di cogliere le motivazioni che hanno determinato, per esempio, integrazioni ${ }^{211}$, quasi sempre utili per meglio chiarire e giustificare, ma soltanto in certi lemmi; la cancellazione di qualche lemma; l'immissione di nuovi lemmi. E il tutto, senza una qualche giustificazione preliminare ${ }^{212}$ e senza un'indicazione ${ }^{213}$, che distingua quanto è traduzione del testo originale e quanto è un'aggiunta recente. La stessa osservazione si deve fare per le note.

Ma passiamo all'esemplificazione.

Per esempio, non avremmo soppresso senza alcuna motivazione le note 1 e 2 di p. 14 dell'originale 214 . La prima, perché ipotizzava ${ }^{215}$, non senza qualche ragione, un errore del $T h L L^{216}$ a proposito della frequenza, davvero troppo bassa (30 volte), di un verbo come ambulo, in uno scrittore come Celso. La seconda, perché non ci sembra un'assurdità - ed è l'unico motivo che noi pensiamo che possa averne motivata l'esclusione - vedere, nel trapasso semantico da 'girare intorno' a 'camminare', una qualche influenza del gr. $\pi \varepsilon \rho \iota \pi \alpha \tau \hat{\omega}$ e della scuola peripatetica ${ }^{217}$, come sembrano confermare esempii del tipo: "finem fecimus et ambulandi et disputandi"218, "cum ambulandi causa in Lyceum uenissemus "219, "ad id aut sedens aut ambulans disputabam "220.

Se l'aggiunta della parola "immorior"221 a lemma viene a correggere un semplice refuso del testo spagnolo e quella del lemma "natis, si veda puga" è nulla piú di un semplice e comodo richiamo, la soppressione del lemma iecur ${ }^{222}$ e l'immissione dei due nuovi "popellus" e "scruta" avrebbero richiesto una qualche segnalazione, se non spiegazione. Cosí, come già abbiamo detto ${ }^{223}$, dovevano essere chiuse tra parentesi 
quadre more solito tutte le integrazioni, per lo piú brevi, ma sempre utili e spesso importanti, volte a migliorare il valore scientifico e la fruibilità dell'opera ${ }^{224}$.

Inspiegabile almeno per noi resta la motivazione di una scelta editoriale che ha fatto sí che soltanto a qualche raro lemma sia stata completata l'esemplificazione ${ }^{225} \mathrm{e}$, peggio ancora, soltanto ad un lemma sia stata aggiunta almeno l'indicazione dei passi in cui la parola compare 226 . Tutti gli altri invece sono rimasti purtroppo com'erano nell'originale 227 .

Difficile è anche capire perché, come già nell'originale, la parola latina, con cui o si apre il discorso o lo si riprende dopo il punto fermo, viene scritta con l'iniziale minuscola 228 e non, com'è usuale, con la maiuscola.

Inoltre, per le parole ossitone che lo richiedono, si sarebbe dovuto usare l'accento o grave o acuto a seconda che la vocale sia o aperta o chiusa e non seguire le (scorrette) norme tipografiche. Se non altro perché $G$. Bonfante è da sempre che si batte sia con l'esempio della sua scrittura, sia in sede teorica - perché questo tipo di accentazione entri nell'uso grafico italiano (cfr., almeno, BONFANTE 1987; 1994b).

C) Si sa che, nonostante tutta l'attenzione, il refuso è sempre in agguato e non manca quasi mai di fare capolino in ogni lavoro. Tuttavia qui, la presenza di errori di stampa è tale da procurare fastidio nel lettore, anche se non giunge mai a limitargli o a impedirgli la comprensione del testo 229 .

\section{Note}

A proposito di: BONFANTE 1994.

BONFANTE 1994b, p. 7.

BONFANTE 1937b.

BONFANTE 1936; 1936b; 1937.

"(Non ricordo bene: credo che fosse il carissimo Dámaso Alonso [...])" (BONFANTE 1994, p. 8).

"Quindici copie non sono, credo, una vera 'edizione" " (BONFANTE 1994, p. 8).

BONFANTE 1994, p. 8.

7 La traduzione dallo spagnolo è opera di M. Vaquero Piñeiro. Per una valutazione cfr. APPENDICE

8 BONFANTE 1994.

9 Perché "lingua parlata" invece dell'originario "elementos populares"? Come scrive nella Prefazione N. Horsfall, 'lingua parlata" "non accontenterà tutti gli specialisti nel campo, dove imperversa da anni un dibattito nient'affatto meramente formale o tecnico sulla terminologia appropriata" (HORSFALL p. 15). E basta citare, in questa sede, almeno RICOTTILLI ( in particolare pp. 36-69). D'altra parte, prosegue N. Horsfall, “'popolare' $[. .$.$] ci portava in una specie di buco nero della lessicografia tra (I) l'uso artistico di elementi tratti dalla$ lingua parlata, (II) il sermo urbanus, cioè il latino informale della gente colta, l'Umgangssprache in senso stretto e corretto [...] e (III) il latino, p. es., dei graffiti pompeiani [...] ed i legami di quel registro lessicale con alcuni nostri testi letterari" (Ibidem).

Come si vede, dunque, N. Horsfall motiva in negativo piuttosto che in positivo la sua scelta, che per noi resta, comunque, arbitraria, anche perché nel testo si è continuato, inspiegabilmente, ad usare l'aggettivo "popolare". Per questo, nella nostra nota, abbiamo preferito mantenere la forma originaria. Cfr., per una simile posizione, TRAINA, p. 244.

10 BONFANTE 1994, p. 17.

11 Ibidem. E forse questo latinista inglese non aveva tutti i torti, se pensiamo che la versione italiana ha già avuto, per quello che è di nostra conoscenza, le recensioni di A. TRAINA, di K. FREUDENBERG, di A. SHARON, di R. MALTBY, di E. LEONOTTI. 
Almeno per evitare l'inconveniente in cui è caduto A. Stefenelli (STEFENELLI) che, come rileva lo stesso G. Bonfante "non conosce i miei Elementos [...] e gli viene così, senza dubbio in buona fede, di ripetere spesso quasi parola per parola quel che avevo detto io 24 anni prima di lui" (BONFANTE 1968, p. 24 n. 5 [= BONFANTE 1987b, p. 535 n. 5]).

13 BONFANTE 1994, p. 26.

14 Ibidem.

15 Ibidem.

16 Cfr., p. es., TRAINA e CIANCAGLNI.

$17 \mathrm{E}$ bene ha fatto $\mathrm{N}$. Horsfall a rimarcare che in nuce la teoria esposta nei primi due capitoli di un libro come quello di B. Axelson (AXELSON) ancora indispensabile per chi opera nel variegato e infido campo della lingua, che soltanto per comodità chiameremo genericamente "non letteraria", c'è già nell'ultimo capitolo di Los elementos" (HORSFALL, p. 17).

18 WÖLFFLIN 1882, p. 85.

19 Cfr. nn. 22, 23.

20 Cfr. BONFANTE 1994, sopra tutto l'Introduzione (pp. 23-26) e la Conclusione (pp. 145-149).

21 BONFANTE 1994, p. 24.

22 Cfr. CIANCAGLINI, p. 138. A sostegno di questa sua valutazione, C. Ciancaglini cita il caso di bracchium (Ibidem), ritenuto da G. Bonfante (BONFANTE 1994, pp. 46-47) popolare per la presenza di $a$ e espressivo a causa di -cc-, mentre "oggi si sa che nel grecismo bracchium ( $\beta p \alpha \chi i \omega v$ ) la resa della aspirata greca con la ge-minata è soltanto indice di trafila osca" (CIANCAGLINI, p. 138). E ciò sul fondamento di quanto affermato in LEUMANN (pp. 250-251) e ripreso e sviluppato in MANCINI (pp. 71-74 e 83-85). Ma questa è una risposta, ma non inoppugnabile ("oggi si sa"); infatti non convince altri studiosi (cfr. HOFMANN 1940 p. 54, ORIOLES, p. $782 \mathrm{n}$. 3). Inoltre la proposta di Bonfante non esclude "con esto, desde luego, el origen griego de la palabra", come si legge in una nota dell' edizione originale (BONFANTE 1937b, p. 25 n. 1), stranamente sostituita nell' edizione italiana con un' assurda (perché, chi l' ha mai detto?) e avulsa (perché, che cosa c' entra?): "In greco non c' è la doppia $c$ "

23 Le citazioni delle parole o dei passi di Orazio sono riprese da BONFANTE 1994, ma verificate su BO1965 e su BORZSÁK. I dati statistici, invece, sono sempre confrontati con quelli che emergono dallo sfoglio degli Indices verborum o delle concordanze, che in piú di mezzo secolo dalla pubblicazione del primo articolo di G. Bonfante, hanno visto la luce. Quando questi strumenti mancano, si accettano per buoni quelli elaborati dall'Autore sui dati forniti dal $T h L L$.

24 Un elenco si può trovare in BONFANTE 1994, Bibliografia, pp. 27-30.

25 Cfr. almeno WÖLFFLIN 1876; 1882; 1884; 1887.

26 RUCKDESCHEL. Un lavoro, che pur condotto con un metodo molto diverso dal suo, anche G. Bonfante giudica fondamentale tanto da puntualizzare che, "chi voglia avere un'idea esauriente degli elementi popolari nella lingua di Orazio dovrà sempre consultare, oltre al mio lavoro, l'eccellente opera di Ruckdeschel" (BONFANTE 1994, p. 25).

27 BOURCIEZ. Studio che, però, "merita la dura recensione di A. Meillet, in BSL, XXVIII, 1928, pp. 133 e ss." (BONFANTE 1994, pp. 25)

28 BONFANTE 1994.

29 Cfr., p. es., n. 24.

30 Cfr. BONFANTE 1994, Indice delle parole e delle espressioni citate, pp. 163-165, in cui si troveranno i rimandi.

31 Cfr. almeno WÖLFFLIN 1876; 1882; 1884.

32 Tra le quali, in primo piano, le Epistulae ad Atticum.

33 Per quanto riguarda Virgilio è però doveroso ricordare la differenza molto marcata tra la lingua piú familiare delle Bucoliche e, sopra tutto, dell'Appendix e quella piú aulica dell'Eneide. Le Georgiche, com'è noto, occupano un posto intermedio.

34 "Respondit Cornelius Tacitus eloquentissime et quod eximium orationi eius inest, $\sigma \varepsilon \mu \nu \omega \varsigma$ " ( PLIN. epist., II, $11,17)$.

35 "Procul grauitate coepti operis crediderim" (TAC., II, 50).

36 NORDEN, I, p. 331.

37 BONFANTE 1994, p. 145. 
39 I testi di riferimento sono BORZSÁK e BO1965.

40 Cfr. BONFANTE 1994, pp. 36-38 (che riporta anche il composto perambulo ${ }^{-}$). Quando è a disposizione il Lexicon o l'Index uerborum di un autore, è sempre stata fatta, da parte nostra, la verifica del dato statistico riportato in BONFANTE 1994. In caso contrario abbiamo considerato buono quello presentato da G. Bonfante.

Diciamo subito, però, che quando abbiamo rilevato qualche discrepanza, questa non è mai stata di tale entità da inficiare il valore delle sue conclusioni.

41 HOR., sat. I, 2, 25; I, 4, 51; I, 4, 66.

42 HOR., ep. IV, 5 ; V, 71; VIII, 14.

43 BONFANTE 1994, p. 37. Cfr. anche ThLL, I, s.u., col. 1870, 1.74, e quanto riportato in ERNOUT-MEILLET, s.u.

44 Cfr. BERKOWITZ-BRUNNER, s.u. (Lucilio), FASCIANO, s.u. (Virgilio); DEFERRARI-FANNING-SULLIVAN, s.u. (Lucano); SCHULTE, s.u. (Valerio Flacco); YOUNG, s.u. (Silio Italico); DEFERRARIEAGAN, s.u. (Stazio).

45 Cfr., rispettivamente, BIRCH, s.u. e RAPSCH-NAJOCK-NOWOSAD, s.u.

46 Cfr. BLACKMAN-BETTS, s.u.

47 Cfr. PACKARD, s.u.

48 Cfr. DEFERRARI-BARRY-McGUIRE, s.u.

49 Cfr. BETTS-ASHWORTH,s.u.

50 Cfr. SCHMEISSER, s.u.

51 Non: "disprezzare" com'è stato tradotto lo sp. despreciar. Per le osservazioni relative agli errori di traduzione, ai refusi, alle integrazioni e soppressioni rispetto all'originale, cfr. APPENDICE.

52 BONFANTE 1994, p. 37.

53 Almeno per quanto detto a p. 5.

54 Cfr. LODGE, s.u. (Plauto); MERGUET 1877-1884, I, s.u. (Cicerone, Orazioni), MERGUET 1887-1894, I, s.u. (Cicerone, Opere filosofiche), OLDFATHER-CANTER-ABBOTT, s.u. (Cicerone, Lettere); ThLL, I, s.u., col. 1870 (Celso); BUSA-ZAMPOLLI, s.u. (Seneca); SCHNEIDER, s.u. (Plinio il Vecchio).

Sulla base dei dati forniti dalle concordanze, non è possibile che "la frequenza della parola in Cicerone si [spieghi] in parte per il grande uso che ne ha fatto nelle Lettere" (BONFANTE 1994, p. 37).

BONFANTE 1994, p. 37.

56 Cfr. OLDFATHER-CANTER-ABBOT, s.u.

57 Cfr. supra, $\mathrm{p} 4$.

58 Cfr. MERGUET 1887-1894, I, s.u.

59 Cfr. MERGUET 1887- 1884, I, s.u.

60 Comunque, sia detto per inciso, nei Vangeli, ambulare ha già il significato di 'camminare', 'andare' (cff., per es., Matteo 11,5: claudi ambulat; Luca 5,23 surge et ambula) ed ha una frequenza molto alta anche presso gli scrittori cristiani, specialmente - com'è ovvio - in senso figurato.

61 BONFANTE 1994, p. 38.

Che su questo verbo tutto non fosse e non sia ancora chiaro è attestato dall'attenzione che gli studiosi gli hanno riservato (cfr. almeno MEYER-LÜBKE, s.u. ambulare; BONFANTE 1955; 1955b; 1963-64; 1975; MAŃCZAK e, recentemente, PROSDOCIMI e ALINEI, p. 30.

62 HOR., sat. I, 10,4 .

63 Cfr. LODGE, s.u.

64 Cfr. BRIGGS 1983, s.u. (Catone); McCARREN, s.u. (Catullo); MARGEUROTH-NAJOCK, s.u. (poeti priapei); KORN-REITZER, s.u. (Petronio); BETTS-ASHWORTH, s.u. (Columella); DUBROCARD, s.u. (Giovenale).

Non sembra, quindi, che la sua presenza possa definirsi "frequente", come annota G. Bonfante (BONFANTE 1994, p. 75).

66 Cfr. BUSA-ZAMPOLLI, s.u.

67 Cfr. SCHNEIDER, s.u.

68 P. es., il rapporto tra ambüläre e inambŭlāre è di questo tipo. Non è, infatti, un caso che in Tito Livio il primo verbo compaia una volta soltanto mentre il secondo sei. Cfr. PACKARD, s.uu. 
Cfr., p. es., defricō (HOWARD-JACKSON, s.u.).

71 Cfr., p. es., perfricō (BONNELL, s.u.).

72 Cfr., p. es., in Ovidio refricō (DEFERRARI-BARRY-McGUIRE s.u.). Non abbiamo invece riscontrato in GOVAERTS la presenza di affricō, che G. Bonfante (BONFANTE 1994, p. 75) cita come appartenente al lessico di Tibullo.

73 L'equivalenza di terére e fricāre risulta in modo netto, crediamo, dai passi citati da G. Bonfante (BONFANTE 1994, p. 75): contritis arbore costis (LUCIL., IX, 32); quod [cacatum] tu si manibus teras fricesque (CATULL., XXIII, 22); fricat arbore costas (VERG., III, 256), il quale, tra l'altro, è l'unico esempio in Virgilio. Questi confronti non convincono, invece, A. Traina (TRAINA, p. 248), che "dal fatto che Virgilio contro l'unico frico delle Georgiche abbia 11 tero e Plauto 12 tero contro 5 frico" (Ibidem) trae la conclusione "che tero è il termine comune e frico quello 'volgare' " (Ibidem).

74 Per la verità, anche terëre, almeno in origine "steht im sexuellen Sinn" (GOLDBERGER, p. 107) e con un valore osceno è usato nel gioco di parole plautino: "Boius est, boiam terit" (PLAUT., Capt. 888). La stessa opinione compare in MONTERO CARTELLE, p. 161 n. 14.

75 Soltanto il composto conterère sopravvive nello sp. curtir e nel port. cortir (cfr. BONFANTE 1994, p. 75 n. 47). Anche il MEYER-LÜBKE, s.u. presenta, citando il DIEZ, s.u., questa proposta. Il COROMINAS, s.u. curtir, giudica invece questo termine "de origen incierto".

Cfr. rom. freca; it. fregare; friul. freá; fr. frayer; prov., cat., sp., port. fregar. Cfr. MEYER-LÜBKE, s.u. frícäre.

Cfr. BONFANTE 1994, p. 78. Cfr., però, anche p. 77.

78 HOR., carm. II, 7, 28.

79 HOR., carm. IV, 12, 28.

80 Cfr. i rispettivi lessici: RAPSCH-NAJOCK-NOWOSAD, s.u.; BIRCH, s.u.; PACKARD, s.u.; BLACKMANN-BETTS, s.u.

81 LODGE, s.u.

82 MARGUET 1887-1894, I, s.u.; OLDFATHER-CANTER-ABBOT, s.u.

83 DUBROCARD, s.u.

84 ThLL, V/1, s.u.

85 BUSA-ZAMPOLLI, s.u.

86 ThLL, V/1, s.u.

87 OLDFATHER-CANTER-PERRY, s.u.

88 ROBERTS, s.u.

89 Come, p. es., Minucio Felice (cfr. KYTZLER-NAJOCK, s.u.), Cipriano (cfr. BOUET-FLEURY-GOULONZUINGHEDAU, s.u.), Lattanzio (cfr. ThLL, V/l, s.u.).

90 HOR., sat. II, 3, 47 e II, 3, 211; HOR., epist. I, 20, 3; HOR., carm. IV, 12, 28.

91 BONFANTE 1994, p. 78.

92 Cfr. BONFANTE 1994, p. 77

93 HOR., epist. I, 2, 14 e I, 12, 20.

94 Ma soltanto nelle Opere filosofiche, cfr. MERGUET 1887-1894, I, s.u.

95 Cfr. ROBERTS, s.u. delirat.

96 Cfr. BONFANTE 1994, pp. 61-65.

97 HOR., epist. I, 7, 19 e I, 15, 40.

98 BONFANTE 1994, p. 61.

99 Cfr. LODGE, s.u. comedo.

100 Il primo numero indica la frequenza di comědēre, il secondo di edēre .

101 Cfr. McGLYN, s.u.

102 I dati riguardano soltanto le Satire Menippee (cfr. RIESE, Index verborum, s.u.) e sono ancora piú convincenti di quelli (4 contro 3) riportati in BONFANTE 1994, p. 61. Nella Vita dei campi, invece, si registra soltanto un comédère rispetto a 8 edère e non a 11 come compare in BONFANTE 1994, p. 62 (cfr. BRIGGS 1983b, s.uu.). 
103 Cfr. MERGUET 1877-1884, I, s.u.; 1887-1894, I, II, s.u.; OLDFATHER-CANTER-ABBOTT, s.u.; ABBOT-OLDFATHER-CANTER, s.u.

104 I dati in BONFANTE 1994, p. 62 sono di poco superiori a quelli da noi riscontrati.

105 Cfr. BUSA-ZAMPOLLI, s.u.

106 Cfr. BETTS-ASHWORTH, s.u.

107 Cfr. KORN-REITZER, s.u.

108 Cfr. CLAESSON, s.u. Dunque, non 7 volte, di cui 3 loci biblici, come riporta G. Bonfante (BONFANTE 1994, p. 62) nella "tavola presentata dal ThLL, V, 100" (BONFANTE 1994, p. 61), dove, per altro, non compare. Comunque, è evidente a tutti che il valore statistico del dato è pressoché uguale.

109 Cfr. CLAESSON, s.u. Anche manduco(r) (18 volte, di cui 11 loci biblici) "appare con un significato molto vicino a edo" (BONFANTE 1994, p. 63).

110 I dati sono quelli di BONFANTE 1994, p. 64. Ma cfr. anche DUTRIPON, $s . u$.

111 Cfr. almeno BEYER; ERNOUT-MEILLET, s.u.; WALDE-HOFMANN, s.u.

112 "Nos in essendo panem et palmulas gustauimus" (SVET., aug., 76, 4-5).

113 “Dum lectica ex regia domum redeo, panis unciam cum paucis acinis unae duracinae comedi" (SVET., aug., 76, 6-8).

114 "Qui in balineo demum post horam primam noctis duas buccas manducaui" (SVET., aug., 76, 10-11).

115 “[...] uescebaturque et ante cenam" (SVET., aug., 76, 2-3).

116 BONFANTE 1994, p. 63.

117 Sp. e port. comer.

118 Cat. ant. manuger; oggi: menjar < fr. manger.

119 Fr. manger. "Forse non è troppo azzardato sostenere che il manduco della [Itala] Lugdunensis prepara il fr. manger", scrive con insolita prudenza G. Bonfante (BONFANTE 1994, p. 63). Noi non abbiamo dubbii. Proprio la presenza in quest'opera di mandūcāre, rispetto al comédère della Vulgata, è un indizio sicuro del trionfo precoce di mandūcāre in Gallia.

120 Log. ant. mandikari (oggi: man(d)igare), che "viene certo dall'italiano" (BONFANTE 1994, p. 63).

121 It. mangiare, che sembra incontestabilmente derivare dal fr. manger, anche se non si sa bene spiegarne il perché, se non ricorrendo alla proposta, di certo non decisiva, di C. Beyer, secondo cui "mangiare mag mit der Ritterkultur als "vornehmes' Wort" (BEYER, p. 28). Mangiare ha poi soppiantato l'antico mandicare, manicare (cfr. l'it. manicaretto), com'è ben evidenziato in Dante, che ha tutte le tre forme: l'it., cioè tosc. (fior. ant.) manicare (Inf., XXXIII, 60), il latinismo manducare (Inf., XXXII, 127), il francesismo mangiare (Inf., XXXII, 134). Per tutto il problema, cfr. BEYER, GRÖBER.

122 Vegl. manonka; rom. mänînc.

123 Cfr. BONFANTE 1994; p. 74.

124 HOR., epist. I, 2, 29; I, 4, 15; 1, 18, 7.

125 HOR., carm. I, 28, 13.

126 Cfr. RAPSCH-NAJOCK-NOWOSAD, s.u. (Sallustio); BIRCH, s.u. (Cesare); BLACKMANN-BETTS, s.u. (Tacito); MERGUET 1877-1884; 1887-1894, OLDFATHER-CANTER-ABBOT, s.u. (Cicerone); GOVAERTS, s.u. (Tibullo); FASCIANO, s.u. (Virgilio); ROBERTS, s.u. (Lucrezio); McGLYN, s.u. (Terenzio).

127 Cfr. DUBROCARD, s.u. (5 volte).

128 Cfr. DE FERRARI-BARRY-McGUIRE, s.u. (11 volte).

129 Ma soltanto nelle opere filosofiche. Cfr. BUSA-ZAMPOLLI, s.u.

130 Cfr. SIEDSCHLAG, s.u. (12 volte).

131 Cfr. OLDFATHER-CANTER-PERRY, s.u. $(9$ volte, che diventano 13 con le forme che compaiono nell'apparato critico).

132 Cfr. DUTRIPON, s.u. (24 volte).

133 Il ThLL, IV, s.u., col. 1578 dà una frequenza di 171 volte (il 71 in BONFANTE 1994 è chiaramente un refuso).

134 Cfr. SCHNEIDER, s.u. (134 volte).

135 Cfr. sic. cuti 'cote', piem. $k u$ 's.s.'. Cfr. MEYER-LÜBKE, s.u.

${ }^{136} \mathrm{~S}$ 'incontra ben 10 volte anche in uno scrittore purista come Cesare. $\mathrm{Cfr}$. BIRCH, s.u. 
137 Cfr. rom. piele, vegl. pial, friul. piel, it. pelle, log. pedde, prov. pel, fr. peau, cat. pell, sp. piel, port. pelle. Cfr. MEYER-LÜBKE, s.u.

138 Cfr. BONFANTE 1994, p. 60.

139 Che, per la verità, soltanto parzialmente si può considerare sinonimo di uocare.

140 Cfr., rispettivamente, BO1965, s.u.; BIRCH, s.u.; RAPSCH-NAJOCK-NOWOSAD, s.u.; BLACKMANNBETTS, s.u.

141 Cfr. LODGE, s.u. (23 volte).

142 Cfr. DEFERRARI-EAGAN, s.u. (31 volte).

143 Cfr. DEFERRARI-BARRY-McGUIRE, s.u. (68 volte).

144 Cfr. VAN KATWIJK, s.u. (27 volte, quindi di due unità appena superiore al dato riportato in BONFANTE 1994, p. 60, che riprende quello del ThLL, III, s.u., col. 1250).

145 Secondo quanto si legge nel ThLL (Ibidem).

145 HOR, sat., I, 1, 12; I, 2, 130; II, 3, 130.

146 HOR., epist. I, 16, 36.

147 Quest'uso è rimasto nelle lingue romanze. Cfr., p. es., il lat. (ma v. nota precedente) si clamet [aliquem] furem, che continua nell'it. se qualcuno mi chiama ladro e nello sp. si alguien me llama ladro.

148 Cfr. rom. chema, vegl. klamuar, friul. clamâ, fr. ant. clamer, it. chiamare, sp. clamar, port. chamar. Cfr. MEYER-LÜBKE, s.u.

149 Cfr. BONFANTE 1994, p̀. 69-70.

150 Cfr., rispettivamente, BIRCH, s.u.; MERGUET 1877-1884; 1887-1894, s.u. e OLDFATHER-CANTERABBOT, s.u.

151 Cfr. LODGE, s.u. ( 20 attestazioni).

152 Cfr. ThLL, IV, s.u., col. 1172.

153 Crepare viene a "sostituire 'morire' con il significato di 'scoppiare'" (BONFANTE 1994, p. 70), a cominciare dagli scrittori cristiani tardi (cfr. ThLL, IV, s.u., col. 1173, 1.31 sgg.). Questo significato, però, come giustamente osserva G. Bonfante, è sicuramente "antico come dimostra decrepitus, che appare già in Plauto [cfr. LODGE, s.u.: 5 volte]" (BONFANTE 1994, p. 70). Su questo problema, cfr. anche RUCKDESCHEL, pp. 76 sgg.; BOURCIEZ, p. 25; GOLDBERGER, pp. 117, 135.

154 HOR., sat. II , 3, 33; HOR., epist. I, 7, 84.

155 HOR., carm. I, 18, 5; II, 17, 26.

156 HOR., epod. 16, 48; HOR., ars., 247.

157 Cfr., rispettivamente, FASCIANO, s.u.; BO1967, s.u.

158 Cfr. DEFERRARI-EAGAN, s.u.

159 Cfr., rispettivamente, DEFERRARI-FANNING-SULLIVAN, s.u.; SCHMEISSER, s.u.

160 Ancora oggi, nelle lingue romanze, le continuazioni del lat. crěpāre sono parole di uso poco raffinato (cfr. sp. e port. quebrar, rom. crěpa), se non decisamente volgari (cfr. it. crepare, fr. crever).

161 Cfr. BONFANTE 1994, pp. 133-134.

162 Cfr., rispettivamente, BIRCH, s.u.; RAPSCH-NAJOCK-NOWOSAD, s.u.; MERGUET 1877-1884, 18871894, s.u.; BLACKMANN-BETTS s.u.

163 Cfr. LODGE, s.u.

164 Cfr. OLDFATHER-CANTER-ABBOTT, s.u.

165 Cfr. BUSA-ZAMPOLLI, s.u.

166 Cfr. KORN-REITZER, s.u.

167 Cfr. BETTS-ASHWORTH, s.u.

168 Cfr. EICHERT, s.u.

169 Cfr. DEFERRARI-BARRY-McGUIRE, s.u.

170 In Virgilio e Silio Italico 5 volte (cfr. WACHT, s.u. e YOUNG, s.u.), 2 volte in Lucano (cfr. DEFERRARI-FANNING-SULLIVAN, s.u.) e una in Stazio (cfr. KLECKA, s.u.).

${ }^{171}$ Una volta (cfr. DUTRIPON, s.u.).

172 Secondo quanto riporta BONFANTE 1994, p. 133, su indicazione, crediamo, del FORCELLINI. Tre occorrenze troviamo in Vartone (cfr. BRIGGS, s.u.; RIESE, Index Verborun, s.u.). 
173 HOR., epist. I, 19, 41.

174 HOR., carm. II, 15, 9; III, 19, 25; IV, 3, 11.

175 Cfr. friul. espes, it. spesso, log. ispissu, fr. épais, prov. espes, sp. espeso, port. espesso. Cfr. MEYER-LÜBKE, s.u.

176 Cfr. rom. des, vegl. dais. Cfr. MEYER-LÜBKE, s.u.

177 Cfr. BONFANTE 1994, pp. 95-98.

178 "In generale, mi sembra che nei buoni classici magnus si usi per una moltitudine di persone (legio, turba, populus), per cose inanimate o concetti astratti (domus, flumen, gaudium, insula, labor, mare, nauis, oppidum, periculum), grandis, invece, per le piante, gli animali e gli uomini" (BONFANTE 1994, p. 95). Grandis, dunque, "significherebbe esattamente 'cresciuto', 'adulto' " (BONFANTE 1994, p. 95, n. 72), come sembra confermare anche il passo virgiliano: "grandiaque effossis mirabitur ossa sepulchris" (VERG., I, 497).

179 Cfr.BIRCH, I, s.u.; e, dunque, non 4, com'è riportato in BONFANTE 1994, p. 97. citata (Ibidem),

Ibidem, però, sono riportati anche 7 passi del Bellum Africanum che, come ha fatto notare G. Bonfante, illuminano il nostro problema. Infatti, "Cesare e l'autore del Bellum Alexandrinum usano magnus dove il Bellum Africanum usa grandis" (BONFANTE 1994, p. 97 n. 80), dal che il "carattere volgare del Bellum Africanum risulta chiaro" (Ibidem).

180 Cfr. BLACKMANN-BETTS, s.u.

181 "La frequenza straordinaria di grandis in Cicerone, si spiega in parte con il fatto che usa grandis (per la prima volta) come termine tecnico per designare lo stile $\alpha \delta$ jóø (23 volte)" (BONFANTE 1994, p. 97 n. 79).

182 Cfr. MARGUET 1877-1884, II, s.u.

183 Cfr. MARGUET 1887-1894, II, s.u.

184 Cfr. WACHT, I, s.u.

185 Cfr. LODGE, s.u.

186 Cfr. SIEDSCHLAG, s.u.

187 E non 4, com'è riportato in BONFANTE 1994, p. 96. Cfr. BO1967, s.u.

188 Cfr. DUBROCARD, s.u.

189 Magnus, infatti, si continua soltanto nell'otrant. maño 'bello'; logud. mannu; prov. manh, sp. ant. maño. Grandis, invece, si afferma in tutto il resto dell'area romanza: it. grande; fr. grand; prov., cat. gran; sp., port. grande. Importante, come ci dice G. Bonfante, il dato del logudorese, perché "ci ricorda che, al tempo della colonizzazione della Sardegna, nel latino volgare, magnus prevaleva ancora su grandis" (BONFANTE 1994, p. 98 n. 82).

190 HOR., sat. I, 4, 50.

191 HOR., sat. I, 6, 3-4.

192 Si tiene conto di tutti i passi e quindi anche di quelli in cui non sarebbe stata possibile la scelta, perché un termine veniva ad indicare un tratto semantico che l'altro non copriva.

193 Cfr. BO1965, s.u.

194 Cfr. BONFANTE 1994, pp. 51-52.

195 Manca soltanto in Cesare; ma, ciò, potrebbe essere imputabile al caso.

196 Cfr. rom. cînta; vegl. kantuor; it. e log. cantare; friul. chantá; fr. chanter; prov., cat., sp., port. cantar. Cfr. MEYER-LÜBKE, s.u.

197 Cfr. LODGE, s.u.

198 Cfr. BO1967, s.u.

199 Cfr. BO1965, s.u.

200 HOR. sat. I, 10, 42-43.

201 HOR. sat. I, 3, 2.

202 QUINT., X, 1, 93.

203 Per rendere schematico, com'è naturale, il nostro discorso, raggrupperemo sotto A) i rilievi sulla traduzione; sotto $B$ ) quelli relativi alle scelte editoriali; sotto $C$ ) i veri e proprii refusi.

204 Cfr. TRAINA; SHARON; FREUDENBERG; MALTBY; LEONOTTI.

205 Cfr. TRAINA.

206 Cfr. TRAINA, p. 246. 
207 Cfr. TRAINA, pp. 244-245. A quelli da lui rilevati, bisogna aggiungere: le concordanze di Livio non sono di 'Packer', ma di 'Packard' (p. 10, r. 5); delle concordanze di Ovidio c'è una riproduzione anastatica: Hildesheim: G. Olms Verlagsbuchhandlung, 1968 (p. 10, r. 6); non 'Torino', ma 'Alessandria' (p. 11, r. 1); insieme al ben noto volume di J.N. Adams avremmo citato quello di E. Montero Cartelle (MONTERO CARTELLE). Un'ultima considerazione, per finire, sulle indicazioni bibliografiche. Pur nel rispetto della volontà di $N$. Horsfall "di imitare l'estrema sobrietà del testo originario" e del principio nobilissimo che cosi facendo si "risparmia tempo, spazio ed alberi" (HORSFALL, p. 9), noi avremmo citato l'opera di H. Quellet (QUELLET) o tra i nuovi strumenti di lavoro o aggiornando la n. 2 di p. 26.

208 Cfr. TRAINA, p. 249 n. 1 e p. 251 n. 1.

209 Cfr. TRAINA, p. 245.

210 Cfr. TRAINA, p. 245 n. 3.

211 Mentre una verifica andava fatta a proposito dell'affermazione: "ho trattato questo problema nella mia comunicazione L'italiano è il latino dell'Urbe" (p. 42). Il problema cui si riferisce è quello relativo alla conservazione di bellus e alla scomparsa invece di pulcher. Ebbene di ciò non si parla affatto nella comunicazione citata (che, tra l'altro, è stata presentata al IIl Congresso Internazionale dei Linguisti [non: di Linguistica]) e non si precisa che è stata poi pubblicata negli Atti (cfr. BONFANTE 1935). Per contro, in essa s.incontra qualche osservazione su cunnus, ma all'omonimo lemma non si fa menzione di questo dato bibliografico.

212 Da questa osservazione sono esclusi, com'è naturale, quegli interventi mirati a restaurare l'ordine alfabetico corretto delle parole ed espressioni studiate, come nel caso di alter = alius e cesso, che nell'edizione spagnola erano in fondo (cfr. BONFANTE 1937b, p. 129). Detto questo, non riusciamo a comprendere perché non si sia data la giusta collocazione anche al lemma - ĕrunt [non - erunt!] (= desinenza del perfetto), che, tra l'altro, in questa forma compare, seppure non nel giusto ordine, nell'Indice delle parole e delle espressioni citate (pp. 163-165), invece di lasciarlo all'ultimo posto, registrato con la dicitura La desinenza del perfetto ěrunt [non -erunt!].

213 Convenzionalmente, ogni aggiunta al testo originale si chiude tra parentesi quadre.

214 Cfr. BONFANTE 1937 b.

215 Restiamo nel campo delle ipotesi, perché non esistono ancora, a tutt'oggi, né le concordanze né un Index verborum di Celso.

216 Cfr. ThLL, I, s.u., col. 1874 r. 23.

217 Cfr. supra, p. 7.

218 CIC., fin., II, 119.

219 CIC., diuin., I, 8 .

$220 \mathrm{CIC}$., tusc., I, 7. Tutti questi esempii sono riportati in BONFANTE 1937b, p. 14, n. 2.

221 I lemmi sono citati cosí: in tondo e tra virgolette quelli di BONFANTE 1994; in corsivo quelli di BONFANTE $1937 \mathrm{~b}$.

222 Al quale lemma, per altro, si rinvia a p. 45 r. 5 !

223 Cfr. n. 210.

224 Non facciamo esempii, perché sono davvero tanti e, tutto sommato, il citarli non ha tutta quella necessità che sarebbe richiesta nel caso che si trattasse di soppressioni.

225 Cfr. "caldior", "chorda", "crustulum", "curtus".

226 Cfr. "cerebrum".

${ }^{227}$ E cioè pochi, con la semplice indicazione dei passi e molti, senza né questa né, ovviamente, l'esemplificazione.

228 Che a p. 108 r. 25 il periodo cominci con una parola latina ad iniziale maiuscola (Mingo) è, come ci dicevano le nostre maestre, l'eccezione che conferma la regola.

229 Ce ne sono di ogni tipo, di cui noi daremo, naturalmente, soltanto una campionatura. A) Corsivo per tondo e viceversa: 'engad.' per 'engad.' (p. 68 r. 23), 'Mer.' per 'Mer.' (p. 99 r. 36), 'bestia' per 'bestia' (p. 118 r. 35); nelle citazioni dell'edizione spagnola il lemma è indicato con il tondo maiuscolo, mentre per quella italiana si è scelto di utilizzare il corsivo maiuscolo, fatta eccezione per un manipolo di lemmi (da "accedo" a "camera", pp. 35-51), che compaiono in tondo maiuscolo. B) Mancanza della quantità: 'blatterare' per 'blatterāre' e 'blattire' per 'blattïre' (p. 46 r. 8), 'a' per 'a' (p. 45 r. 29, p. 68 r. 27), 'frigidus' per 'frigidus' (p. 51 r. 6); anche nell'Indice delle parole e delle espressioni citate (pp. 161-165), molte vocali sono prive della quantità: 'cesso' per 'cessō', -erunt per -ěrunt, puga per pāga. C) Errori nell'ortografia italiana: 'sostituizione' per 'sostituzione' (p. 50 r. 1), 'da' per 
'dà' (p. 64 r. 35) e latina: 'vulgariter' per 'uulgariter' (p. 47 r. 20), 'NVMMATVUM' per 'NVMMATVM' (p. 43 r. 3), 'cogn-/omen' per 'cogno-/men' (p. 69 rr. 16-17); nei nomi degli autori: 'Ruckedeschel' per 'Ruckdeschel' (p. 86 r. 28); nelle parole straniere modeme: '[spagn.] ladro' per 'ladrón' (p. 60 r. 16), '[spagn.] porqué' per 'porque' (p. 72 r. 26), 'got. fraîtan' per 'got. *fraitan' (p. 61 r. 13), 'port. escuitar' per 'escutar' (p. 41 r. 29), 'engad. brac' per 'engad. brač' (p. 47 r. 7). Dove, però, si registra una percentuale davvero inusitata di errori è nelle citazioni di parole romene: 'umblà' per 'umbla' (p. 38 r. 4), 'ascultà' per 'asculta' (p. 41 r. 31 ), 'brat' per 'braț' (p. 47 r. 8), 'catà' per 'căta' (p. 53 r. 14), 'crepà' per 'crěpa' (p. 70 r. 6).

\section{Bibliografia citata}

ALINEI: M. Alinei, Etimologie nel cassetto, "Quaderni della Sezione di Glottologia e Linguistica del Dipartimento di Studi Medievali e Moderni dell'Università degli Studi 'G. D'Annunzio' di Chieti”, 7 (1995), pp. 13-37.

ABBOTT-OLDFATHER-CANTER: K. M. Abbott - W. A. Oldfather - H. V. Canter, Index Verborum in Ciceronis Rhetorica, necnon incerti auctoris libros Ad Herennium, with the assistence of R. F. Butler - L. M. Kaiser - M. B. Tehon-L. Palmer Zickgraf, based on the editions of Cicero's Rhetorica by A. S. Wilkins, De inventione by E. Stroebel, and the second edition of Ad Herennium by F. Marx, Urbana: University of Illinois Press, 1964.

AXELSON: B. Axelson, Unpoetische Wörter. Ein Beitrag zur kenntnis der lateinischen Dichtersprache, Lund: Gleerup, 1945.

BERKOWITZ-BRUNNER: L. B. Berkowitz - Th. F. Brunner, Index Lucilianus, Hildesheim: G. Olms Verlagsbuchhandlung, 1968.

BETTS-ASHWORTH: C. G. Betts - W. D. Ashworth, Index to the Uppsala edition of Columella, Uppsala: Almqvistand Wicksells, 1971.

BEYER: C. Beyer, Die Verba des 'Essens', 'Schickens', 'Kaufens' und 'Eindens' in ihrer Geschichte von Latein bis in die romanischen Sprachen, Leipzig : Selbstverlag des Romanischen Seminars - Paris: Librairie E. Droz, 1934.

BIRCH, Concordantia et Index Caesaris / Concordance and Index to Caesar, edited by C. M. Birch, HildesheimZürich-New York: Olms-Weidmann, 1989, voll. I-II.

BLACKMANN-BETTS: Concordantia Tacitea / A concordance to Tacitus, edited by D. R. Blackmann - G. C. Betts, Hildesheim-Zürich-New York: Olms-Weid-mann, 1986, voll. I-II.

BO 1965: D. Bo, Lexikon Horatianum, Hildesheim: G. Olms Verlagsbuchhandlung, 1965, I/1 (A-K)-I / 2 (L-Z).

BO 1967: D. Bo, Auli Persii Flacci Lexicon, Hildesheim: G. Olms Verlagsbuchhand-lung, 1967.

BONFANTE 1935: G. Bonfante, L'italiano è il latino dell'Urbe, in Atti del III Congresso Internazionale dei Linguisti (Roma, 19-26 settembre 1933), a cura di B. Migliorini e V. Pisani, Firenze: Felice Le Monnier, 1935, pp.316-319.

BONFANTE 1936: G. Bonfante, Los elementos populares en la lengua de Horacio (primera parte), "Emerita, Boletin de lingüistica y filología clásica", $4 / 1$ (1936), pp. 86-119.

BONFANTE 1936b: G. Bonfante, Los elementos populares en la lengua de Horacio (segunda parte), "Emerita, Boletín de lingüística y filología clásica", 4/2 (1936), pp. 209-247.

BONFANTE 1937: G. Bonfante, Los elementos populares en la lengua de Horacio, "Emerita, Boletin de lingüistica y filología clásica”, 5/1 (1937), pp. 17-88.

BONFANTE 1937b: G. Bonfante, Los elementos populares en la lengua de Horacio, Madrid: Centro de estudios históricos, 1937.

BONFANTE 1955: G. Bonfante, Intorno al verbo andare, "Lingua Nostra", 16/2 (1955), pp. 38-40.

BONFANTE 1955b: G. Bonfante, Ancora andare, "Lingua Nostra", 16/4 (1955), pp. 99-100.

BONFANTE 1963-64: G. Bonfante, $E$ ancora andare!, "Studi linguistici italiani", 4 (1963-64), pp. 160-169.

BONFANTE 1968: G. Bonfante, Quando si è cominciato a parlare italiano?, in Festschrift Walther von Wartburg zum 80. Geburtstag 18. Mai 1968, herausgegeben von K. Baldinger, Tübingen: Max Niemeyer Verlag, 1968, pp. 21-46.

BONFANTE 1975: G. Bonfante, Encore it. andare, "Revue roumaine de linguistique", 20/2 (1975), p. 167. 
BONFANTE 1987: G. Bonfante, Progetto di microriforma dell'ortografia italiana, in BONFANTE 1987b, pp. 555-569.

BONFANTE 1987b: Scritti scelti di Giuliano Bonfante, a cura di R. Gendre. II. Latino e romanzo, Alessandria: Edizioni dell'Orso, 1987.

BONFANTE 1994: G. Bonfante, La lingua parlata in Orazio. Prefazione di N. Horsfall [Premessa di G. Bonfante], Venosa: Edizioni Osanna, 1994.

BONFANTE 1994b: G. Bonfante, Premessa, in BONFANTE 1994, pp. 7-8

BONFANTE 1994c: G. Bonfante, L'ortografia italiana: progetto di miniriforma, "Accademia Nazionale dei Lincei. Classe di Scienze Morali, Storiche e Filologiche. Rendiconti”, s. IX, vol. V/2 (1994), pp. 189-190.

BONNELL: Lexicon Quintilianeum, edidit E. Bonnellus, Hildesheim: Georg Olms Verlagsbuchhandlung, 1962 [ripr. anast. di: Leipzig 1834].

BORZSÁK: Q. Horati Flacci Opera, edidit S. Borzsák, Leipzig: B. G. Teubner Verlagsgesellschaft, 1984.

BOUET-FLEURY-GOULON-ZUINGHEDAU: Cyprien, Traités. Concordance: documentation lexicale et grammaticale, ed. P. Bouet, Ph. Fleury, A. Goulon, M. Zuinghedau, avec la collaboration de P. Dufraigne, Hildesheim-Zürich-New York: Olms-Weidmann, 1986.

BOURCIEZ: J. Bourciez, Le "sermo cotidianus" dans les satires d'Horace, Bordeaux 1927.

BRIGGS 1983: Concordantia in Catonis librum De Agri Cultura, edidit by W. W. Briggs jr., with the technical assistance of T. R. White, Hildesheim-Zürich-New York: Olms-Weidmann, 1983.

BRIGGS 1983b: Concordantia in Varronis libros De Re Rustica, edited by W. W. Briggs jr., with the technical assistence of T. R. White and Ch. G. Shirley jr., Hildesheim-Zürich-New York: Georg Olms Verlag, 1983.

BUSA-ZAMPOLLI, Concordantiae senecanae, curaverunt R. Busa - A. Zampolli, Hildesheim-New York: G. Olms Verlag, 1975, voll. I-II.

CATULL: $v$. SCHUSTER-EISENHUT.

CHARPIN: Lucilius, Satires, texte établi, traduit et annoté par F. Charpin, Paris: Les Belles Lettres, 1978-1991, voll. I (1978) - II (1979) - III (1991).

CIANCAGLINI: C. Ciancaglini, Bonfante Giuliano, in Enciclopedia oraziana, Roma: Istituto dell' Enciclopedia Italiana, 1998, III, pp. 137-138.

CIC.: v. MERGUET 1877-1884; MERGUET 1877-1894; OLDFATHER-CANTER-ABBOTT.

CLAESSON: G. Claesson, Index Tertullianeus, Paris: Etudes Augustiniennes, 1974-1975, voll. 3 [A-E (1974); FP (1975); Q-Z (1975)].

COROMINAS: J. Corominas, Breve diccionario etimológico de la lengua castellana, Madrid: Editorial Gredos, $1967^{2}$.

DEFERRARI-BARRY-McGUIRE: R. J. Deferrari - M. I. Barry - M. R. P. McGuire, A concordance of Ovid, Washington: The Catholic University of America press, 1939.

DEFERRARI-FANNING-SULLIVAN: R. J. Deferrari - M. W. Fanning - A. S. Sullivan, A concordance of Lucan, Hildesheim: G. Olms Verlagsbuchhandlung, 1965.

DIEZ: F. Diez, Etymologisches Wörterbuch der romanischen Sprachen. Mit einem Anhang von A. Scheler, Bonn: $1878^{4}$.

DUBROCARD: M. Dubrocard, Juvenal-Satires, Index verborum. Relevés statistiques, avec la collaboration du Laboratoire d'Analyse statistique des langues anciennes de l'Université de Liège (Professeur Delatte), Hildesheim-New York: G. Olms Verlag, 1976.

DUTRIPON: F. P. Dutripon, Bibliorum Sacrorum Concordantiae, Hildesheim-New York: Georg Olms Verlag, 1976.

EICHERT: O. Eichert, Vollständiges Wörterbuch zu dem Geschichtswerke des Quintus Curtius Rufus, Hannover: Han'sche Buchhandlung, 1893.

ERNOUT-MEILLET: E. Ernout - A. Meillet, Dictionnaire étymologique de la langue latine. Histoire des mots, Paris: Librairie C. Klincksieck, 19674.

FORCELLINI: Lexicon totius latinitatis ab Ae. Forcellini [...] lucubratum [...] a I. Furlanetto $[\ldots]$ emendatum et auctum nunc vero curantibus F. Corradini et I. Perin [...] emendatius et auctius melioremque in formam redactum, Typis seminarii Patavii, 1940, voll. I-IV.

FREUDENBURG: $\mathrm{K}$. Freudenburg, On dressing well in a dead language. A review of $\mathrm{G}$. Bonfante, La lingua parlata in Orazio (Venosa, 1994), "Arachnion. A Journal of ancient literature and history on the Web", 3 (1995), pp. 4 [= http://www.cisi.unito.it/arachne/num3/freudenb.html]. 
GOLDBERGER: W. Goldberger, Kraftausdrücke im Vulgärlatein, "Glotta", 20 (1932), pp. 101-150.

GOVAERTS: S. Govaerts, Le corpus Tibullianum. Index verborum et Relevés statistiques. Essai de méthodologie statistique, La Haye: Mouton and Co, 1966.

GRÖBER: G. Gröber, Vulgärlateinische Substrata romanischer Wörter, in Archiv für lateinische Lexikographie und Grammatik mit Einschluss des älteren Mittellateins [...], herausgegeben von E. Wölfflin, Leipzig: Druck und Verlag von B.G.Teubner, 1886, vol. 3, pp. 507-531.

HOFMANN: 1940: J. B. Hofmann, Altitalische Sprachdenkmäler 1931-1937, "Bursian Jahresbericht". 270 (1940), pp. 1-122

HOFMANN: J .B. Hofmann, La lingua d'uso latina, introduzione, traduzione italiana e note, a cura di L. Ricottilli, Bologna: Patron Editore, 19852 [19801].

HOR.: v. BORZSÁK.

HORSFALL: N. Horsfall, Prefazione, in BONFANTE 1994, pp. 9-19.

HOWARD-JACKSON: Index uerborum C. Suetoni Tranquilli stilistique eius proprietatum nonnullarum. Confecerunt A. A. Howard - C. N. Jackson, Hildesheim: G. Olms, 1963 [ripr. anast. di: Cantabrigiae Massachusettensium, 1922].

IHM: C. Svetoni Tranquilli Opera, I: De vita Caesarum libri VIII, recensuit M. Ihm, Stutgardiae in saedibus B. G. Teubneri, 1967 [rist. anast. di: 1958].

KLECKA: Concordantia in Publium Papinium Statium, ed. J. Klecka, Hildesheim-Zürich-New York: Olms Verlag, 1983.

KORN-REITZER: M. Korn - S. Reitzer, Concordantia Petroniana. Computerkon-kordanz zu den Satyrica des Petron, Hildesheim-Zürich-New York: Olms-Weidmann, 1986.

KYTZLER-NAJOCK: Concordantia in Minuci Felicis Octavium, curantibus B. Kytzler et D. Najock adiuvante A. Nowosad, Hildesheim-Zürich-New York: Olms-Weidmann, 1991.

LEONOTTI: E. Leonotti, rec. a G. Bonfante, La lingua parlata in Orazio, Ed. Osanna, Venosa, 1994, pp. 168, $£$. 20.000, “Aevum", 71/1 (1997), pp.192-194.

LEUMANN: M. Leumann, Literaturbericht für das Jahr 1927, "Glotta", 18 (1930), pp. 2.41-274

LODGE: Lexicon Plautinum conscripsit G. Lodge, Hildesheim: G. Olms Verlagsbuchhandlung, 1962, voll. I-II [rist. anast. di: Leipzig 19241].

LUCIL.: v. CHARPIN.

MCCARREN: A critical concordance to Catullus, edited by V.P. McCarren, with the computer assistence of W. Tajibnapis, Leiden: E. J. Brill, 1977.

McGLYNN: P. McGlyn, Lexicon Terentianum conscripsit P. McGlynn olim in litteris humanioribus praelector in Universitate Glasguensi, In aedibus Blackie et Fili Londini et Glasguae, 1963-1967, voll. I: A-O (1936); II: P-V (1967).

MALTBY: R. Maltby, rec. a G. Bonfante, La lingua parlata in Orazio. Traduzione dallo spagnolo di M. Vaquero Piñeiro. Prefazione di N. Horsfall (first published in Spanish in 1937). Pp. XXXI+165. Venosa: Edizioni Osanna, 1994. Paper, £. 20.000, "The Classical Review", n.s. 45/2 (1995), p. 443.

MANCINI: M. Mancini, Aspirate greche e geminate latine, 'Biblioteca di Ricerche Linguistiche e Filologiche [del Dipartimento di Studi Glottoantropologici dell' Università di Roma « La Sapienza »]. 28', Viterbo: Istituto di Studi Ro-manzi. Università della Tuscia, 1990.

MAŃCZAK: W. Mańczak, Une étymologie romane controversée: aller, andar, etc., "Revue Roumaine de Linguistique" 19/2 (1974), pp. 89-101.

MARGEUROTH-NAJOCK: H. Margeuroth - N. Najock, Concordantiae in Corpus Priapeorum et Pervigilium Veneris, Hildesheim: 1983.

MERGUET 1877-1884: H. Merguet, Lexicon zu den Reden des Cicero, mit Angabe sämtlicher Stellen, Jena: Verlag von H. Durft, 1877-1884, voll. I (1877), II (1880), III (1882), IV (1884).

MERGUET 1887-1894: H. Merguet, Lexikon zu den philosophischen Schriften Cicero's, mit Angabe sämtlicher Stellen, Jena: Verlag von G. Fischer, 1887-1894, voll. I (1887), II (1892), III (1894).

MEYER-LÜBKE: W. Meyer-Lübke, Romanisches Etymologisches Wörterbuch, Heidelberg: C. Winter Universitätsverlag, $1972^{5}$.

MONTERO CARTELLE: E. Montero Cartelle, El latín erótico. Aspectos léxicos y literarios (hasta el s. I d.C.), Sevilla: Publicaciones de la Universidad de Sevilla, $1991^{2}$. 
NORDEN: E. Norden, La prosa d'arte antica. Dal VI secolo a.C. all'età della Rinascenza. Edizione italiana a cura di B. Heinemann Campana, con una Nota di aggiornamento di G. Calboli e una Premessa di S. Mariotti, Roma: Salerno, s.d. [ma 1986], [trad. di: Leipzig 1898].

OLDFATHER-CANTER-ABBOTT: W. A. Oldfather - H. V. Canter - K. M. Abbott, Index Verborum Ciceronis Epistularum, based on the edition by L. C. Pursez in Oxford Classical Texts (1901-1903), with the assistance of friends and students and in completion of the task inaugurated by M. N. Westmore and A. M. Dame, Urbana: University of Illinois Press, 1938.

OLDFATHER-CANTER-PERRY: W. A. Oldfather - H. V. Canter-B. E. Perry, Index Apuleianus, with the assistance of K. M. Abbot and others friends and former students, Middletown, Conn.: The American Philological Association, 1934.

ORIOLES: V. Orioles, Mediazione osca e interferenze greco-latine, "Incontri Linguistici", 2 (1975), pp. 177-191.

PACKARD: D. W. Packard, A concordance to Livy, Cambridge, Mass.: Harvard University Press: 1968, voll. I (A-D), II (E-I).

PLIN.: v. SCHUSTER-HANSLIK.

PROSDOCIMI: A. L. Prosdocimi, Italiano 'andare', in Omaggio a G. Folena, Padova, 1993, III, pp. 2479-2432.

QUELLET: H. Quellet, Bibliographia indicum, lexicorum et concordantiarum auctorum Latinorum / Répertoire bibliographique des index, lexiques et concordances des auteurs latins, Hildesheim-New York: Georg Olms Verlag, 1980.

QUINT.: v. RADERMACHER.

RADERMACHER: $M$. Fabi Quintiliani Iustitutionis oratoriae libri XII, edidit L. Radermacher. Pars prior libros I-VI continens. Pars secunda libros VII-XII continens. Editio stereotypa correctior editionis primae. Addenda et corrigenda collegit et adiecit V. Buchheit, Lipsiae in aedibus B. G. Teubneri, 1959.

RAPSCH-NAJOCK-NOWISAD: Concordantia in Corpus Sallustianum, Hildesheim-Zürich-New York: OlmsWeidmann, 1991, voll.I-II.

RICOTTILLI: L. Ricottilli, Introduzione: Hofmann e il concetto di lingua d'uso, in HOFMANN, pp. 9-69.

RIESE: M. T. Varro, Saturarum Menippearum reliquiae, recensuit, prolegomena scripsit, appendicem adiecit A. Riese, Hildesheim-New York: Georg Olms Verlag, 1971 [rist. anast. di: Leipzig, 1865].

ROBERTS: L. Roberts, A concordance of Lucretius, New York-London: Garland publishing Inc., 1977.

RUCKDESCHEL: F. Ruckdeschel, Archaismen und Vulgarismen in der Sprache des Horaz, Erlanden: Meucke, 1911.

SCHMEISSER: B. Schmeisser, A concordance to the Elegies of Propertius, Hildesheim: Verlag dr. H.A. Gerstenberg, 1972.

SCHNEIDER: In C. Plini Secundi Naturalis Historiae libros indices, composuit O. Schneider, Hildesheim: G. Olms Verlagsbuchhandlung, 1967, voll. I (A-L), II (U-Z).

SCHUSTER-EISENHUT: Catulli Veronensis liber, recensuit M. Schuster editionem stereotypam correctionem. Editionis secundae curavit W. Eisenhut, Lipsiae in aedibus B. G. Teubneri, 1958.

SCHUSTER-HANSLIK: C. Plini Caecili Secundi Epistularum libri novem, Epistularum ad Traianum liber, Panegyricus, recensuit M. Schuster et R. Hanslik, Lipsiae in aedibus B. G. Teubneri, 1958.

SHARON: A. Sharon, rec. a G. Bonfante, La lingua parlata in Orazio. Venosa: Edizioni Osanna Venosa, 1994. Pp. 165 (paperback), "The Classical Bulletin", 71 (1995), pp. 51-52.

SIEDSCHLAG: E. Siedschlag, Martial-Konkordanz, Hildesheim: G. Olms Verlag, 1967.

STEFENELLI: A. Stefenelli, Die Volkssprache im Werk des Petron (im Hinblick auf die romanischen Sprachen), Wien: Braumüller, 1962.

SVET.: v. IHM

TAC.: v. WELLESLEY

ThLL: Thesaurus Linguae Latinae, editus auctoritate et consilio Academicarum quinque Germanicanam [...], Lipsiae in aedibus B. G. Teubneri.

TRAINA: A. Traina, 'Parole' in Orazio, "Rivista di filologia e d'istruzione classica", 122/2 (1994), pp. $244-252$.

VAN KATWIJK: Lexicon Commodianeum, cum introductione de Commodiani vita, temporibus, sermone [...], A. F. Van Katwwijk [...], Amstelodami apud M. J. Portielje, 1934.

VERG.: Virgile, Les Géorgiques, texte établi et traduit par H. Goelzer, Paris: Société d'édition "Les Belles Lettres", $1947^{5}$ 
WACHT: Concordantia Vergiliana curavit M. Wacht, Hildesheim-Zürich-New York: Olms-Weidmann, 1966, voll. I: A-L; II: M-Z.

WALDE-HOFMANN: A. Walde, Lateinisches etymologisches Wörterbuch. 3., neubearbeitete Auflage von J. B. Hofmann, Heidelberg: Carl Winter's Universitätsbuchhandlung, 1938-1954, voll. I (A-L) - II (U-Z).

WELLESLEY: C. Taciti libri qui supersunt, ediderunt S. Borzsák et K. Wellesley. II/1: Historiarum libri, edidit K. Wellesley, Leipzig: B. G. Teubner Verlagsgesellschaft, 1989.

WÖLFFLIN 1876: E. Wölfflin, Bemerkungen zum Vulgärlatein, "Phil." 34 (1876) 137.

WÖLFFLIN 1882: E. Wölfflin, Ueber die Aufgaben der lat. Lexikographie, "Rheinische Museum", 37 (1882), pp. 83-123.

WÖLFFLIN 1884: E. Wölfflin, Vorwort, in Archiv für lateinische Lexikographie und Grammatik mit Einschluss des älteren Mittellateins [...] herausgegeben von E. Wölfflin, Leipzig: Druck und Verlag von B. G. Teubner, 1884, vol. I, pp. 1-20.

WÖLFFLIN 1887: E. Wölfflin, Lateinische und romanische Komparation, Erlangen, 1887.

YOUNG: N.D.Young, Index verborum Silianus, 'Iowa studies in classical philology' $n^{\circ} 8$, Iowa City, Ia: Athens Press, 1939.

\section{Povzetek}

\section{PRVINE LJUDSKEGA JEZIKA PRI HORACIJ}

Vir za analizo metode avtorjevega dela je Bonfantejeva študija La lingua parlata in Orazio, Venosa, 1994, kar je italijanski prevod izvirne objave v španščini (Madrid, 1937). Pri tem se je upoštevalo gradivo, ki ga je Giuliano Bonfante uporabil, seveda ob kritičnem tehtanju njegovih ugotovitev in $z$ željo, da se, kadar je to potrebno, izboljšajo ali natančneje postavijo leksikografska in metodološka vprašanja.

Tehtanje prvin ljudskega in celo družinskega jezika Satir in Epistol omogoča živo predstavo vsakdana antičnega Rima; še več, omogoča oceniti, kako Horacijeva poezija prihaja do tistega tako težko dosegljivega cilja: umetnosti enostavnega. 